\title{
A NOTE ON THE HOMOMORPHISM THEOREM FOR HEMIRINGS
}

\author{
D. M. OLSON \\ Department of Mathematics \\ Cameron University \\ Lawton, Oklahoma 73501 \\ U.S.A.
}

(Recieved April 11, 1978)

ABSTRACT. The fundamental homomorphism theorem for rings is not generally applicable in hemiring theory. In this paper, we show that for the class of $\mathrm{N}$-homomorphism of hemirings the fundamental theorem is valid. In addition, the concept of $\mathrm{N}$-homomorphism is used to prove that every hereditarily semisubtractive hemiring is of type (K).

KEY WORDS AND PHRASES. Hemirings, homomorphism of hemirings, homomorphism Theorem, N-homorphism, Type $(K)$ ideals, Hereditarily semisubtractive. 
1. INTRODUCTION.

It is well known that the analogue to the fundamental homomorphism theorem is not necessarily true in general hemiring theory. However, in [1] Allen defined a class of maximal homomorphisms of hemirings for which the exact analogue could be proven. In this article we extend his class to the class of N-homomorphisms for which the homomorphism theorem holds, and examine some properties of $\mathrm{N}$-homomorphisms .

Also, in [2] LaTorre defines the concepts of a hemiring being of type (H) or type (K). He gives results establishing certain classes of hemirings as being all of type (H), but states that no general statement can be made concerning the occurance of hemiring of type $(K)$. In section 4 we use the concept of an N-homomorphism together with the idea of a semisubtractive hemiring [4] to establish that all hereditarily semisubtractive hemirings are of type (K).

In what follows we use the standard hemiring definitions and terminology which may be found in [1] and [2].

\section{N-HOMOMORPHISMS.}

DEFINITION 1. A hemiring homomorphism $\phi$ from $S$ onto $T$ is called a maximal homomorphism if for every $t \varepsilon T$, there exists $c_{t} \varepsilon \phi^{-1}(t)$ such that for all $x \in \phi^{-1}(t)$ we have $x+\operatorname{ker} \phi \subseteq c_{t}+\operatorname{ker} \phi$. [1]

DEFINITION 2. A hemiring homomorphism $\phi$ from $S$ onto $T$ is called an

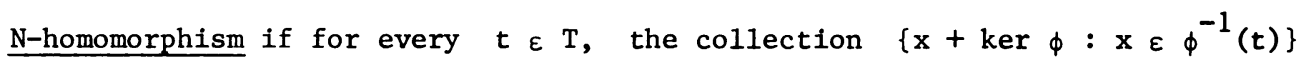
contains no two sets which are disjoint.

It is easy to see that $\phi: \mathrm{S} \rightarrow \mathrm{T}$ will be an $\mathrm{N}$-homomorphism if and only if whenever $\phi(x)=\phi(y)$ for some $x, y \in S$ we have $k_{1}, k_{2} \varepsilon$ ker $\phi$ such that $x+k_{1}=y+k_{2}$. LaTorre [3] has also characterized maxiaml homomorphism as follows. 
LEMMA 3. A homomorphism $\phi: S \rightarrow T$ is maximal if and only if the inverse image of every $t \varepsilon T$ is a coset of ker $\phi$.

With this lemma we can establish the following result.

THEOREM 4. If $\phi: \mathrm{S} \rightarrow \mathrm{T}$ is a maximal homomorphism then $\phi$ is an $\mathrm{N}-$ homomorphism.

PROOF. Let $\phi: S \rightarrow T$ be a maximal homomorphism and let $t \varepsilon T$. Now consider $x+\operatorname{ker} \phi$ and $y+\operatorname{ker} \phi$ for $x, y \varepsilon \phi^{-1}(t)$. By Lemma $1, \phi^{-1}(t)$ is a coset of $\operatorname{ker} \phi$, say $\phi^{-1}(t)=c+\operatorname{ker} \phi$. Then $x, y \varepsilon c+\operatorname{ker} \phi$. Let $\mathrm{x}=\mathrm{c}+\mathrm{k}_{1}$, and $\mathrm{y}=\mathrm{c}+\mathrm{k}_{2}$, then $\mathrm{x}+\mathrm{k}_{2}=\mathrm{y}+\mathrm{k}_{1}$ for $\mathrm{k}_{1}, \mathrm{k}_{2} \varepsilon$ ker $\phi$ and $\mathrm{x}+\operatorname{ker} \phi \cap \mathrm{y}+\operatorname{ker} \phi \neq \phi$. Since $\mathrm{t}, \mathrm{x}$ and $\mathrm{y}$ are arbitrary we see that $\phi$ is an $\mathrm{N}$-homomorphism.

Since every ring homomorphism is maximal, these are also N-homomorphisms. In addition it is easy to verify that every natural may of a hemiring $S$ onto a quotient hemiring $\mathrm{S} / \mathrm{I}$ is an $\mathrm{N}$-homomorphism. The following example shows that the class of $\mathrm{N}$-homomorphisms does not coincide with the class of maximal homomorphisms.

EXAMPLE 5. Let $S=\{0,1,2,3,4\}$ be the hemiring with zero multiplication and addition defined by the following table.

\begin{tabular}{l|lllll} 
& 0 & 1 & 2 & 3 & 4 \\
\hline 0 & 0 & 1 & 2 & 3 & 4 \\
1 & 1 & 1 & 4 & 4 & 4 \\
2 & 2 & 4 & 4 & 4 & 4 \\
3 & 3 & 4 & 4 & 4 & 4 \\
4 & 4 & 4 & 4 & 4 & 4
\end{tabular}

Let $\mathrm{T}$ be the subhemiring $\{0,1\}$ of $\mathrm{S}$. Define $\phi: \mathrm{S} \rightarrow \mathrm{T}$ by $\phi: 0,1 \rightarrow 0$ 
and $2,3,4 \rightarrow 1$ then $\phi$ is a hemiring homomorphism with $\operatorname{ker} \phi=\{0,1\}$.

Now $\phi^{-1}(1)=\{2,3,4\}$ and no two of $2+\operatorname{ker} \phi, 3+\operatorname{ker} \phi$ and $4+\operatorname{ker} \phi$ are disjoint. Thus $\phi$ is an N-homomorhpism. However, $\phi^{-1}(1)$ is not a coset of $\operatorname{ker} \phi$ so $\phi$ is not a maximal homomorphism.

\section{THE FUNDAMENTAL HOMOMORPHISM THEOREM}

For the class of N-homorphism we can establish analogues to certain desirable results from ring theory.

LEMMA 6. If $\phi$ is an $\mathrm{N}$-homomorphism from $\mathrm{S}$ onto $\mathrm{T}$ with ker $\phi=(0)$, then $\phi$ is an isomorphism.

PROOF. Suppose $\phi(x)=\phi(y)$. Then there exist $k_{1}, k_{2} \varepsilon$ ker $\phi$ such that $x+k_{1}=y+k_{2}$. But since $\operatorname{ker} \phi=(0), k_{1}=k_{2}=0$. Thus $x=y$ and $\phi$ is an isomorphism.

THEOREM 7. If $\phi$ is an N-homomorphism from $S$ onto $T$ then $S /$ ker $\phi \cong T$.

PROOF. Define $\psi:$ S/ker $\phi \rightarrow T$ by $\psi([s])=\phi(s)$, where [s] is the equivalence class of $S$ in $S$ determined by the ideal ker $\phi$ of $S$. Then as usual $\psi$ is a well defined onto homomorphism. If $\psi([s])=\psi([t])$, then $\phi(s)=\phi(t)$ and, since $\phi$ is an $N$-homomorphism, there must exist $k_{1}, k_{2}, \varepsilon$ ker $\phi$ such that $s+k_{1}=t+k_{2}$. But then by definition $[s]=[t]$ and $\psi$ is an isomorphism.

It is clear that the class of N-homomorphism is the largest class for which the mapping $\psi$, as defined in the proof of Theorem 7, will be an isomorphism.

THEOREM 8. If $\phi$ is an N-homomorphism from $S$ onto $T$ and $K$ is an Ideal of $\mathrm{T}$, then $\mathrm{s}_{/}^{\prime} \phi^{-1}(\mathrm{~K}) \cong \mathrm{T} / \mathrm{K}$.

PROOF. Define $\psi: S \rightarrow T / K$ by $\psi(s)=[\phi(s)]$. Then one can quickly check 
to see that $\psi$ is a homomorphism from $S$ onto $T / K$. Now if $\psi(s)=\psi(t)$

then $[\phi(s)]=[\phi(t)]$ which implies that $\phi(s)+k_{1}=\phi(t)+k_{2}$ for some $\mathrm{k}_{1}, \mathrm{k}_{2} \varepsilon \mathrm{k}$. Choose $\ell_{1}$ and $\ell_{2}$ from $\phi^{-1}\left(\mathrm{k}_{1}\right)$ and $\phi^{-1}\left(\mathrm{k}_{2}\right)$ respectively. Then $\phi\left(s+\ell_{1}\right)=\phi\left(t+\ell_{2}\right)$. Since $\phi$ is an N-homomorphism, there exist $z_{1}, z_{2} \varepsilon \operatorname{ker} \phi \subseteq \phi^{-1}(K)$ such that $s+\left(\ell_{1}+z_{1}\right)=t+\left(\ell_{2}+z_{2}\right)$. But since $\ell_{1}+z_{1}, \ell_{2}+z_{2} \varepsilon \phi^{-1}(\mathrm{~K})$, they are both in ker $\psi$ and thus $\psi$ is an N-homomorphism.

$$
\text { Finally } \phi^{-1}(\mathrm{~K})^{*} \subseteq \operatorname{ker} \psi=\{\mathrm{s} \varepsilon \mathrm{S}: \psi(\mathrm{s})=[\phi(\mathrm{s})]=0\}=\left\{\mathrm{s} \varepsilon \mathrm{S}: \phi(\mathrm{s}) \varepsilon \mathrm{K}^{*}\right\} \text {. }
$$

But if $\phi(s) \varepsilon K^{*}$ then $\phi(s)+\phi(k) \varepsilon K$ for some $k \varepsilon \phi^{-1}(K)$. Thus $\mathrm{s}+\mathrm{k} \varepsilon \phi^{-1}(\mathrm{~K})$ so $\mathrm{s} \varepsilon \phi^{-1}(\mathrm{~K})^{*}$. This gives us that $\operatorname{ker} \psi=\phi^{-1}(\mathrm{~K})^{*}$ so by the preceeding theorem $\mathrm{S} / \phi^{-1}(\mathrm{~K})=\mathrm{S} / \phi^{-1}(\mathrm{~K}) * \tilde{\mathrm{T}} / \mathrm{K}$.

\section{HEMIRINGS OF TYPE $\mathrm{K}$}

DEFINITION 9. A hemiring $S$ is of type $(K)$ provided that if $I$ is a $k$-ideal of $S$ and $n: S \rightarrow S / I$ is the natural homomorphism, then $n$ preserves k-ideals. [ 2]

DEFINITION 10. A hemiring $S$ is said to be semisubtractive if for every pair of elements $a$ and $b$ in $S$ at least one of the equations $a+x=b$ or $b+x=a$ is solvable in $S$. [4]

DEFINITION 11. A hemiring $\mathrm{S}$ is hereditarily semisubtractive if each ideal of $S$ is semisubtractive as a hemiring.

Clearly every ring is hereditarily semisubtractive and also the hemiring of non-negative integers under the operations of $a+b=\max \{a, b\}$ and $a \cdot b=\min \{a, b\}$. In view of the following lemma any semisubtractive hemiring whose ideals are all k-ideals is also hereditarily semisubtractive.

LEMMA 12. If $S$ is a semisubtractive hemiring and $K$ is a $k$-ideal of 
$S$ then $\mathrm{K}$ is semisubtractive.

PROOF. Let $\mathrm{a}, \mathrm{b} \in \mathrm{K}$. Since $\mathrm{a}, \mathrm{b} \in \mathrm{S}$, there exists an element $\mathrm{s} \varepsilon \mathrm{S}$ for which either $a+s=b$ or $b+s=a$. For the sake of argument say $a+s=b$. But then $a+s \varepsilon K$ with $a \varepsilon K$ and since $K$ is a k-ideal this requires $s$ to be $K$. Thus $K$ is indeed semisubtractive.

THEOREM 13. If $\mathrm{S}$ is hereditarily semisubtractive and $\phi$ is an N-homomorphism from $S$ onto $T$ then $\phi$ preserves k-ideals.

PROOF. Let $\mathrm{K}$ be a k-ideal of $\mathrm{S}$ and $\overline{\mathbf{X}}=\phi(\mathrm{K})$. We shall show that $\overrightarrow{\mathrm{K}}^{*} \subseteq \overline{\mathrm{K}}$ and thus that $\overline{\mathrm{K}}$ is a k-ideal. We use the notation $\overline{\mathrm{s}}$ for the image of $s$ under $\phi$ whenever it is convenient. If $\bar{x} \varepsilon \bar{k}^{*}$ then $\bar{x}+\bar{k}_{1}=\bar{k}_{2}$ for some $\overline{\mathrm{k}}_{1}, \overline{\mathrm{k}}_{2} \varepsilon \overline{\mathrm{k}}$. Then $\phi\left(\mathrm{x}+\mathrm{k}_{1}\right)=\phi\left(\mathrm{k}_{2}\right)$ so there exist $\mathrm{z}_{1}, \mathrm{z}_{2} \varepsilon$ ker $\phi$ such that $x+k_{1}+z_{1}=k_{2}+z_{2}$. Since $k+\operatorname{ker} \phi \quad$ is semisubtractive as an ideal of $S$ we have either there exists $t \varepsilon K+\operatorname{ker} \phi$ such that $k_{1}+t=z_{2}$ or there exists $t \in K+\operatorname{ker} \phi$ such that $k_{1}=z_{2}+t$.

In the first case we see that $x+k_{1}+t+z_{1}=k_{2}+z_{2}+t$ which implies that $x+z_{2}+z_{1}=k_{2}+z_{2}+t$. Then $\bar{x}=\phi(x)=\phi\left(x+z_{1}+z_{2}\right)=$ $\phi\left(\mathrm{k}_{1}+\mathrm{z}_{2}+\mathrm{t}\right) \varepsilon \phi(\mathrm{K})=\overline{\mathrm{K}}$.

In the xecond case we get $x+k_{1}+z_{1}+t=k_{2}+z_{2}+t$ so $x+k_{1}+z_{1}+t=k_{2}+k_{1}$. Now $t \varepsilon k+k e r \phi$ so $t=k_{3}+z_{3}$ and as a result we have $x+k_{1}+k_{3}+\left(z_{1}+z_{3}\right)=k_{2}+k_{1}$. Since $k$ is a $k$-ideal of $S$ we have $x+z_{1}+z_{3} \varepsilon K$. Then $\bar{x}=\phi\left(x+z_{1}+z_{3}\right) \varepsilon \phi(K)=\bar{K}$. In any case we get $\overline{\mathrm{K}}^{*} \subseteq \overline{\mathrm{K}}$ and so $\phi(\mathrm{K})=\overline{\mathrm{K}}$ is a k-ideal of $\mathrm{T}$ as desired.

COROLLARY 14. If $S$ is an hereditarily semisubtractive hemiring then $S$ is of type $(\mathrm{K})$.

PROOF. If $I$ is a k-ideal of $S$ the natural map $n: S \rightarrow S / I$ is an $N-$ homomorphism. By Theorem $13 \quad n$ preserves k-ideals which makes $S$ a hemiring 
of type (K).

\section{REFERENCES}

1. Allen, Paul J., "A Fundamental Theorem of Homomorphism for Semirings," Proc. Amer. Math. Soc. 21 (1969) 412-416.

2. LaTorre, D. R., "On h-ideals and k-ideals in Hemirimgs," Pub1. Math. Debrecen 12 (1965) 219-226.

3. LaTorre, D. R., "A Note on Quotient Semirings," Proc. Amer. Math. Soc. 24 (1970) 463-465.

4. Mosher, James R., "Generalized Quotients of Hemirings," Compositio Math. 22 (1970) 275-281. 


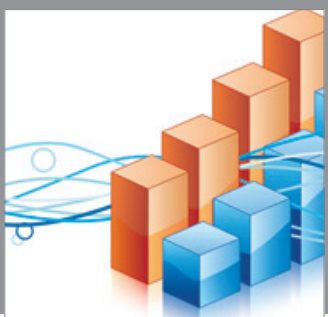

Advances in

Operations Research

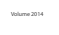

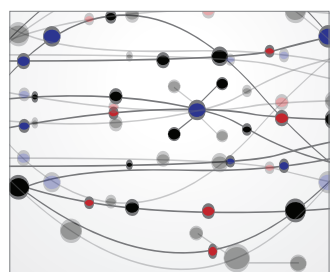

\section{The Scientific} World Journal
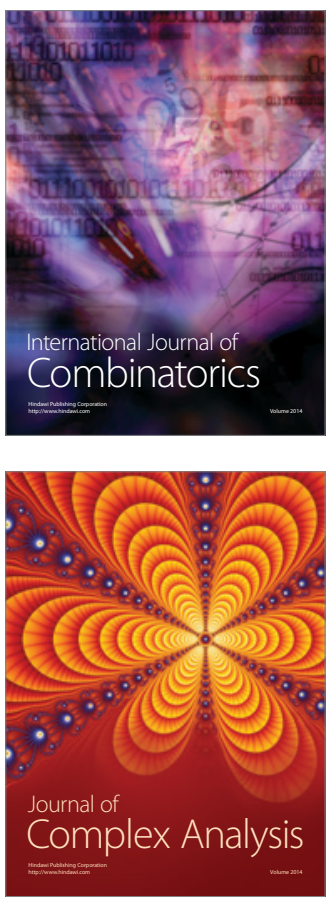

International Journal of

Mathematics and

Mathematical

Sciences
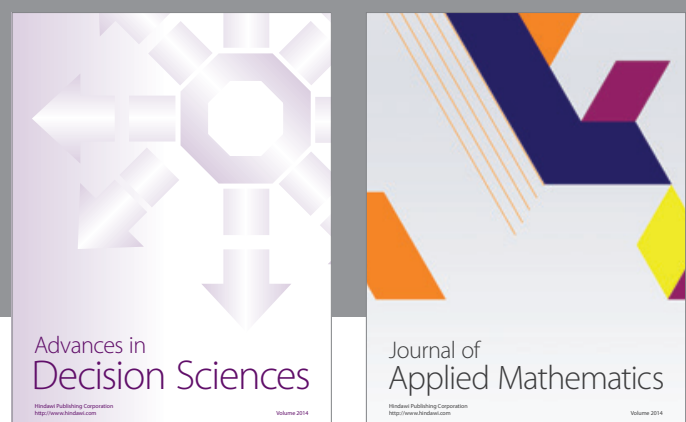

Journal of

Applied Mathematics
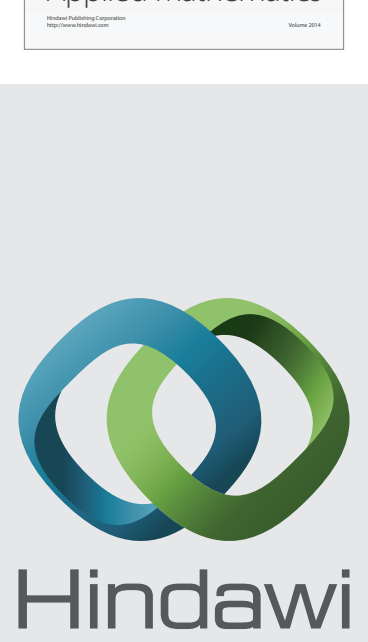

Submit your manuscripts at http://www.hindawi.com
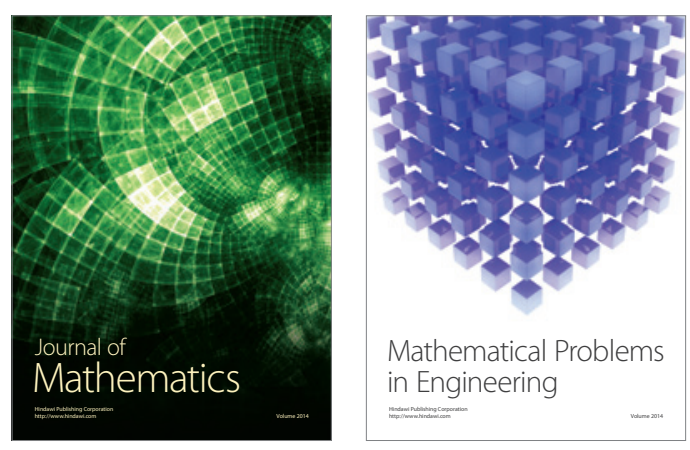

Mathematical Problems in Engineering
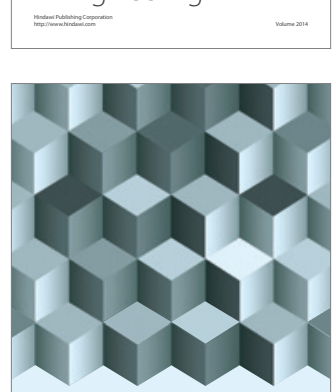

Journal of

Function Spaces
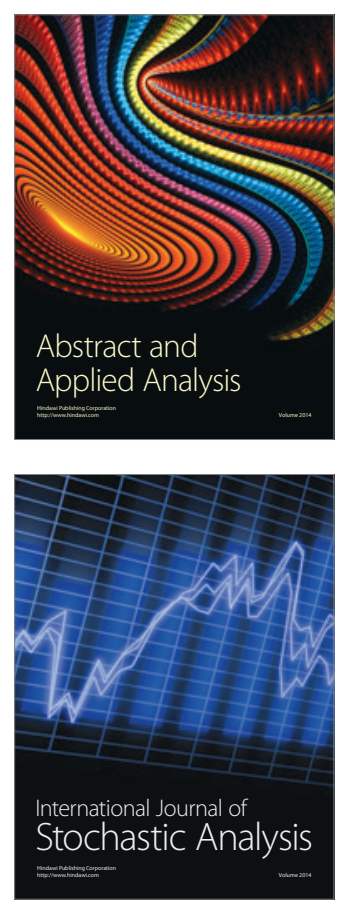

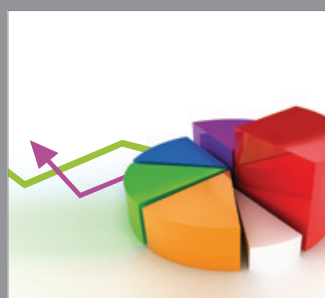

ournal of

Probability and Statistics

Promensencen
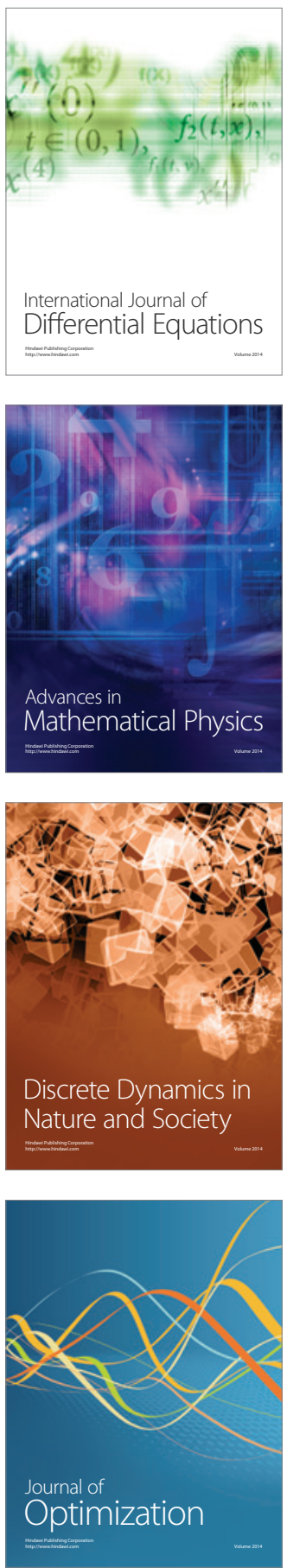